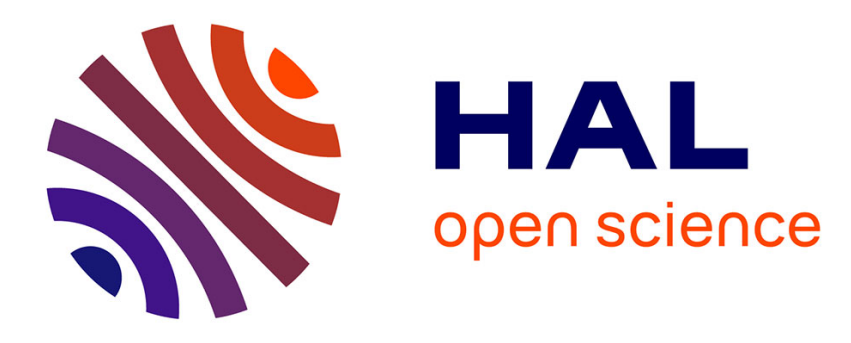

\title{
An Ingham type proof for the boundary observability of a $\mathrm{N}$-d wave equation
}

Michel Mehrenberger

\section{To cite this version:}

Michel Mehrenberger. An Ingham type proof for the boundary observability of a N-d wave equation. Comptes Rendus. Mathématique, 2009, 10.1016/j.crma.2008.11.002 . hal-00311730

\section{HAL Id: hal-00311730 \\ https://hal.science/hal-00311730}

Submitted on 20 Aug 2008

HAL is a multi-disciplinary open access archive for the deposit and dissemination of scientific research documents, whether they are published or not. The documents may come from teaching and research institutions in France or abroad, or from public or private research centers.
L'archive ouverte pluridisciplinaire HAL, est destinée au dépôt et à la diffusion de documents scientifiques de niveau recherche, publiés ou non, émanant des établissements d'enseignement et de recherche français ou étrangers, des laboratoires publics ou privés. 
Optimal Control

\title{
An Ingham type proof for the boundary observability of a $N$-d wave equation
}

\author{
Michel Mehrenberger, \\ Institut de Recherche Mathématique Avancée, Université Louis-Pasteur, UMR CNRS 7501, \\ 7, rue René Descartes, 67084 Strasbourg, France \\ Received $* * * * *$; accepted after revision +++++ \\ Presented by
}

\begin{abstract}
The boundary observability of the wave equation has been studied by many authors. A method of choice is to use the multiplier method (cf [3]). Recently, in [6], a first Fourier based proof is given in the case where the domain is a square, thanks to a new Hautus type test. We give here a new self-contained proof with an Ingham type approach in the more general case where the domain is a product of intervals; this leads, in contrary to the proof in [6], to explicit time and constants. However, we do not reach the optimal time which can be obtained for this problem by the multiplier method. To cite this article: A. Name1, A. Name2, C. R. Acad. Sci. Paris, Ser. I 340 (2005).
\end{abstract}

\section{Résumé}

Une preuve de type Ingham pour l'observabilité frontière d'une équation des ondes $N-d$. L'observabilité frontière de l'équation des ondes a été étudiée par de nombreux auteurs. Une méthode de choix est d'utiliser la méthode des multiplicateurs (cf [3]). Récemment, dans [6], une première preuve basée sur les séries de Fourier a été donnée dans le cas où le domaine est un carré grâce à un test de type Hautus. On donne ici une nouvelle preuve auto-contenue par une approche de type Ingham, dans le cas plus général où le domaine est un produit d'intervalles; on obtient alors un temps et des constantes explicites, contrairement à la preuve de [6]. Cependant, on n'atteint pas le temps optimal, qui peut être obtenu pour ce problème par la méthode des multiplicateurs. Pour citer cet article: A. Name1, A. Name2, C. R. Acad. Sci. Paris, Ser. I 340 (2005).

Email address: mehrenbe@math.u-strasbg.fr (Michel Mehrenberger). 


\section{Version française abrégée}

Soit $N \in \mathbb{N}^{*}$, des réels $\left.a_{i}<b_{i} i=1, \ldots, N, \Omega=\prod_{i=1}^{N}\right] a_{i}, b_{i}$ [ et $u(t, x)$ solution de (1). Le système est bien défini pour des conditions initiales satisfaisant $\left(u_{0}, u_{1}\right) \in H_{0}^{1}(\Omega) \times L^{2}(\Omega)$. On a la proposition classique suivante.

Proposition 0.1 Il existe $T_{0}>0$ tel que pour $T>T_{0}$, le système (1) est observable : il existe une constante $c>0$ telle que l'on ait (2) pour tout $\left(u_{0}, u_{1}\right) \in H_{0}^{1}(\Omega) \times L^{2}(\Omega)$.

La méthode des multiplicateurs (cf. [3]) permet d'obtenir cette inégalité d'observabilité (2) avec un temps optimal $T_{0}=2 \sqrt{\sum_{i=1}^{N}\left(b_{i}-a_{i}\right)^{2}}$.

Dans certaines situations, on peut aussi essayer d'obtenir une preuve utilisant une inégalité d'Ingham (cf [4]). Comme la solution de (1) est explicitement donnée en termes de série de Fourier, une question naturelle est : peut-on obtenir (2) avec des arguments de type Ingham? La difficulté principale, dans le cadre multi-dimensionnel $(N>1)$ est que des valeurs propres en nombre arbitraire peuvent être arbitrairement proches, ce qui rend l'obtention de l'inégalité d'observabilité par une telle approche plus difficile. Notons que de tels cas ont déjà été considérés dans d'autres situations (voir [5], [1]). Certains théorèmes multi-dimensionnels de type Ingham ont été établis et ont permis de traiter par exemple du cas de l'équation des plaques avec contrôle interne [2]. Cependant, à notre connaissance, le cas de l'observabilité frontière de l'équation des ondes en dimension $N>1$ n'a jamais été traité dans la littérature par une approche de type Ingham. Notons que (2) a été prouvé pour le cas du carré à l'aide de séries de Fourier, grâce à un test de type Hautus dans [6], sans temps explicite d'observation. Le but de cette présente Note est de fournir une preuve auto-contenue de type Ingham de (2), dans le cas (déjà précisé) où le domaine est un produit d'intervalles. On utilise d'abord l'orthogonalité des vecteurs propres, puis on applique la première méthode d'Ingham. Grâce à une décomposition convenable, on peut se débarasser des termes où il n'y a pas d'écart (comme dans [5]) et on peut donc obtenir l'inégalité d'observabilité désirée. Le temps d'observation, ainsi que les constantes impliquées dans l'inégalité peuvent être explicitées, mais nous n'avons pas pu atteindre le temps optimal par cette méthode.

\section{Introduction}

Let $N \in \mathbb{N}^{*}$, reals $\left.a_{i}<b_{i} i=1, \ldots, N, \Omega=\prod_{i=1}^{N}\right] a_{i}, b_{i}[$ and $u(t, x)$ solution of

$$
\left\{\begin{array}{l}
u^{\prime \prime}=\Delta u, \quad 0<t<T, \quad x \in \Omega \\
u=0, \quad 0<t<T, \quad x \in \partial \Omega \\
u(0, x)=u_{0}(x), \quad u^{\prime}(0, x)=u_{1}(x), \quad x \in \Omega .
\end{array}\right.
$$

The system is well defined for initial conditions satisfying $\left(u_{0}, u_{1}\right) \in H_{0}^{1}(\Omega) \times L^{2}(\Omega)$. We have the following classical proposition.

Proposition 1.1 Let $\Gamma=\cup_{j=1}^{N}\left(\prod_{i=1}^{j-1}\left[a_{i}, b_{i}\right] \times\left\{a_{j}\right\} \times \prod_{i=j+1}^{N}\left[a_{i}, b_{i}\right]\right)$. There exists $T_{0}>0$ such that for $T>T_{0}$, the system (1) is observable: there exists a constant $c>0$ such that we have

$$
\left\|u_{0}\right\|_{H_{0}^{1}(\Omega)}^{2}+\left\|u_{1}\right\|_{L^{2}(\Omega)}^{2} \leq c \int_{0}^{T} \int_{\Gamma}\left|\partial_{\nu} u(t, x)\right|^{2} \mathrm{~d} \Gamma \mathrm{dt},
$$

for all $\left(u_{0}, u_{1}\right) \in H_{0}^{1}(\Omega) \times L^{2}(\Omega)$.

One famous method to study such an observability inequality is to use the multiplier method (cf [3]). We can here effectively apply this method and obtain (2) with an optimal time $T_{0}=2 \sqrt{\sum_{i=1}^{N}\left(b_{i}-a_{i}\right)^{2}}$. 
In some situations, we can also try to use an Ingham inequality (cf [4]).

The solution of (1) is explicitely given by terms of a Fourier series:

$$
u(t, x)=\sum_{k \in\left(\mathbb{N}^{*}\right)^{N}}\left(c_{k} e^{i \omega_{k} t}+c_{-k} e^{-i \omega_{k} t}\right) \prod_{j=1}^{N} \sin \left(\frac{\pi k_{j}\left(x_{j}-a_{j}\right)}{b_{j}-a_{j}}\right),
$$

with suitable coefficients $c_{k}$, and with the notations

$$
k=\left(k_{1}, \ldots, k_{N}\right), x=\left(x_{1}, \ldots, x_{N}\right), \omega_{k}=\sqrt{\sum_{j=1}^{N}\left(\frac{k_{j} \pi}{b_{j}-a_{j}}\right)^{2}} .
$$

A natural question is then, can we obtain (2) with Ingham type arguments? The main difficulty, in the multi-dimensional setting $(N>1)$, is that arbitrarily many eigenvalues can be arbitrarily close, which makes the obtention of the observability inequality by such an approach more difficult. Note that such cases have already been considered in other situations (see [5], [1]). Some multi-dimensional Ingham type theorems have been stated and enabled to deal with e. g. the case of the plate equation with internal control [2]. However, to our knowledge, the case of the boundary observability of the wave equation has never been considered in the literature by a Ingham type approach. Note that (2) has been proven for the case of the square by means of Fourier series, thanks to a Hautus type test in [6], without an explicit time of observation. The aim of the present note is to provide a simple self contained Ingham type proof of (2), in the case (already precised) where the domain is a product of intervals. We first use the orthogonality of the eigenfunctions, and then apply Ingham's first method. Thanks to a convenient decomposition, we can get rid of the terms where there is no gap (like in [5]) and thus obtain the desired observability inequality. The time of observation, like the constant involved in the inequality can be explicited, but we were not able to reach the optimal time with that method.

In the sequel, we will write $A \asymp B$ instead of $c_{1} A \leq B \leq c_{2} A$ and $A \succsim B$ instead of $A \geq c_{1} B$ for brevity, where $c_{1}, c_{2}>0$ are constants.

\section{A new Ingham inequality}

We present here a new Ingham inequality which will enable us to prove the Proposition 1.1 as an application, and which may have its own interest. It also could be applied for other problems.

Theorem 2.1 Let $d \in \mathbb{N}^{*}$, an infinite set $\mathcal{K} \subset \mathbb{N}^{*}$, reals $\left(\lambda_{k}\right)_{k \in \mathcal{K}^{d}}$, and complexes $\left(p_{\ell}\right)_{\ell \in \mathcal{K} \text {. }}$. We suppose the following gap assumption: for $j=1, \ldots, d$, there exists $\gamma_{j}>0$ such that

$$
\begin{aligned}
& \left|\lambda_{k_{1}, \ldots, k_{j-1}, k_{j}, k_{j+1}, \ldots, k_{d}}-\lambda_{k_{1}, \ldots, k_{j-1}, k_{j}^{\prime}, k_{j+1}, \ldots, k_{d}}\right| \geq \gamma_{j}\left|k_{j}-k_{j}^{\prime}\right|, \\
& \left|\lambda_{k_{1}, \ldots, k_{j-1}, k_{j}, k_{j+1}, \ldots, k_{d}}+\lambda_{k_{1}, \ldots, k_{j-1}, k_{j}^{\prime}, k_{j+1}, \ldots, k_{d}}\right| \geq \gamma_{j}\left|k_{j}+k_{j}^{\prime}\right|,
\end{aligned}
$$

for all the indices $k=\left(k_{1}, \ldots, k_{d}\right) \in \mathcal{K}^{d}$ and $k_{j}^{\prime} \in \mathcal{K}$ such that the weights $\left(p_{\ell}\right)_{\ell \in \mathcal{K}}$ satisfy

$$
\max _{i=1, \ldots, d, i \neq j}\left|p_{k_{i}}\right| \leq \max \left(\left|p_{k_{j}}\right|,\left|p_{k_{j}^{\prime}}\right|\right) .
$$

Then, for $T>2 \pi \sqrt{\sum_{i=1}^{d} \frac{1}{\gamma_{i}^{2}}}$, there exists a constant $c_{1}>0$ such that we have,

$$
\sum_{j=1}^{d} \sum_{k_{1}, \ldots, k_{j-1}, k_{j+1}, \ldots, k_{d} \in \mathcal{K}} \int_{0}^{T}\left|\sum_{k_{j} \in \mathcal{K}} p_{k_{j}}\left(a_{k} e^{i \lambda_{k} t}+a_{-k} e^{-i \lambda_{k} t}\right)\right|^{2} \mathrm{~d} t \geq c_{1} \sum_{k \in \mathcal{K}^{d}}\left(\left|a_{k}\right|^{2}+\left|a_{-k}\right|^{2}\right)\left(\sum_{j=1}^{d}\left|p_{k_{j}}\right|^{2}\right),
$$


for all complexes $\left(a_{k}\right)_{k \in \mathcal{K}^{d}}$ and $\left(a_{-k}\right)_{k \in \mathcal{K}^{d}}$, such that the sums involved are finite.

The proof consists in using Ingham's first method. We recall here the definition and the main properties that will be useful. We consider the function

$$
k(t)= \begin{cases}\cos \frac{\pi t}{T} & \text { if }|t| \leq T / 2 \\ 0 & \text { if }|t|>T / 2 .\end{cases}
$$

It's Fourier transform $\hat{k}$ satisfies: $\hat{k}(x)=\int_{-\infty}^{\infty} k(t) e^{i x t} \mathrm{~d} t=-\frac{2 T \pi \cos (x T / 2)}{x^{2} T^{2}-\pi^{2}}$. We have $\hat{k}(0)=\frac{2 T}{\pi}$, and for $\gamma>\frac{2 \pi}{T}, \ell \in \mathbb{N}^{*}$ and $|x| \geq \ell \gamma$,

$$
|\hat{k}(x)| \leq \frac{2 T}{\pi} \frac{1}{\left|\frac{x^{2} T^{2}}{\pi^{2}}-1\right|}=\frac{2 T}{\pi}\left(\frac{2 \pi}{\gamma T}\right)^{2} \frac{1}{\left|4\left(\frac{x}{\gamma}\right)^{2}-\left(\frac{2 \pi}{\gamma T}\right)^{2}\right|} \leq \frac{2 T}{\pi}\left(\frac{2 \pi}{\gamma T}\right)^{2} \frac{1}{4 \ell^{2}-1} .
$$

We recall also that

$$
\sum_{\ell=1}^{\infty} \frac{1}{4 \ell^{2}-1}=\frac{1}{2} \sum_{\ell=1}^{\infty}\left(\frac{1}{2 \ell-1}-\frac{1}{2 \ell+1}\right)=\frac{1}{2}
$$

We set $b_{k}(t)=a_{k} e^{i \lambda_{k} t}+a_{-k} e^{-i \lambda_{k} t}$. Now, since $k(t) \leq 1_{[-T / 2, T / 2]}$, we have

$$
A:=\int_{-T / 2}^{T / 2}\left|\sum_{k_{j} \in \mathcal{K}} p_{k_{j}} b_{k}(t)\right|^{2} \mathrm{~d} t \geq \int_{\mathbb{R}} k(t)\left|\sum_{k_{j} \in \mathcal{K}} p_{k_{j}} b_{k}(t)\right|^{2} \mathrm{~d} t .
$$

We note $\delta_{j}=\max _{i=1, \ldots, d, i \neq j}\left|p_{k_{i}}\right|$ and then have the decomposition

$$
\begin{aligned}
A \geq \int_{\mathbb{R}} k(t)\left|\sum_{k_{j} \in \mathcal{K}, \delta_{j} \leq\left|p_{k_{j}}\right|} p_{k_{j}} b_{k}(t)+\sum_{k_{j} \in \mathcal{K}, \delta_{j}>\left|p_{k_{j}}\right|} p_{k_{j}} b_{k}(t)\right|^{2} \mathrm{~d} t \\
=\sum_{\left(k_{j}, k_{j}^{\prime}\right) \in \Lambda_{1}}+\sum_{\left(k_{j}, k_{j}^{\prime}\right) \in \Lambda_{2}}+\sum_{\left(k_{j}, k_{j}^{\prime}\right) \in \Lambda_{3}} B_{k}+\int_{\mathbb{R}} k(t)\left|\sum_{k_{j} \in \mathcal{K}, \delta_{j}>\left|p_{k_{j}}\right|} p_{k_{j}} b_{k}(t)\right|^{2} \mathrm{~d} t,
\end{aligned}
$$

by setting for brevity $k^{\prime}=\left(k_{1}, \ldots, k_{j-1}, k_{j}^{\prime}, k_{j+1}, \ldots, k_{d}\right), \Lambda_{1}:=\left\{\left(k_{j}, k_{j}^{\prime}\right) \in \mathcal{K}, \delta_{j} \leq\left|p_{k_{j}}\right|, \delta_{j} \leq\left|p_{k_{j}^{\prime}}\right|\right\}$,

$$
\Lambda_{2}:=\left\{\left(k_{j}, k_{j}^{\prime}\right) \in \mathcal{K}, \delta_{j} \leq\left|p_{k_{j}}\right|, \delta_{j}>\left|p_{k_{j}^{\prime}}\right|\right\}, \Lambda_{3}:=\left\{\left(k_{j}, k_{j}^{\prime}\right) \in \mathcal{K}, \delta_{j} \leq\left|p_{k_{j}}\right|, \delta_{j}>\left|p_{k_{j}^{\prime}}\right|\right\},
$$

and, since $\hat{k}(x)=\hat{k}(-x), B_{k}=p_{k_{j}} \overline{p_{k_{j}^{\prime}}}\left(\left(a_{k} \overline{a_{k^{\prime}}}+a_{-k} \overline{a_{-k^{\prime}}}\right) \hat{k}\left(\lambda_{k}-\lambda_{k^{\prime}}\right)+\left(a_{k} \overline{a_{-k^{\prime}}}+a_{-k} \overline{a_{k^{\prime}}}\right) \hat{k}\left(\lambda_{k}+\lambda_{k^{\prime}}\right)\right)$. The key point here is that we can get rid of the last term of (10) where there is no gap assumption thanks to the positiveness of $k$. This trick was already used in [5] (see also [1]).

For the first term of (10), by using the assumption (4)-(5)-(6), the fact that $T>\frac{2 \pi}{\gamma_{j}}$, the property (8), and since $\hat{k}(0)=\frac{2 T}{\pi}$, we get, by putting $A_{k}=\left|a_{k}\right|^{2}+\left|a_{-k}\right|^{2}$,

$$
\sum_{\left(k_{j}, k_{j}^{\prime}\right) \in \Lambda_{1}} B_{k} \geq \frac{2 T}{\pi}\left(\sum_{k_{j} \in \mathcal{K}, \delta_{j} \leq\left|p_{k_{j}}\right|}\left|p_{k_{j}}\right|^{2} A_{k}-\sum_{\left(k_{j}, k_{j}^{\prime}\right) \in \Lambda_{1}, k_{j} \neq k_{j}^{\prime}} C_{k}\right),
$$

where

$$
C_{k}=\frac{\left|p_{k_{j}}\right|^{2} A_{k}+\left|p_{k_{j}^{\prime}}\right|^{2} A_{k^{\prime}}}{2}\left(\frac{2 \pi}{T \gamma_{j}}\right)^{2}\left(\frac{1}{4\left(k_{j}-k_{j}^{\prime}\right)^{2}-1}+\frac{1}{4\left(k_{j}+k_{j}^{\prime}\right)^{2}-1}\right) .
$$


For the second and the third terms of (10), we obtain similarly

$$
\sum_{\left(k_{j}, k_{j}^{\prime}\right) \in \Lambda_{2}} B_{k} \geq-\frac{2 T}{\pi} \sum_{\left(k_{j}, k_{j}^{\prime}\right) \in \Lambda_{2}} C_{k} \sum_{\left(k_{j}, k_{j}^{\prime}\right) \in \Lambda_{3}} B_{k} \geq-\frac{2 T}{\pi} \sum_{\left(k_{j}, k_{j}^{\prime}\right) \in \Lambda_{3}} C_{k} .
$$

By gathering the estimates of the four terms of (10) and then using (9), we thus get

$$
\frac{\pi}{2 T} A \geq \sum_{k_{j} \in \mathcal{K}, \delta_{j} \leq\left|p_{k_{j}}\right|}\left|p_{k_{j}}\right|^{2}\left|a_{k}\right|^{2}-\sum_{k_{j}, k_{j}^{\prime} \in \mathcal{K}, k_{j} \neq k_{j}^{\prime}} C_{k} \geq \sum_{k_{j} \in \mathcal{K}, \delta_{j} \leq\left|p_{k_{j}}\right|}\left|p_{k_{j}}\right|^{2}\left|a_{k}\right|^{2}-\sum_{k_{j} \in \mathcal{K}}\left|a_{k} p_{k_{j}}\right|^{2}\left(\frac{2 \pi}{T \gamma_{j}}\right)^{2} .
$$

We then obtain, by summing over $j=1, \ldots, d$ and $k \in \mathcal{K}^{d}$,

$$
\begin{aligned}
& B:=\sum_{j=1}^{d} \sum_{k_{1}, \ldots, k_{j-1}, k_{j+1}, \ldots, k_{d} \in \mathcal{K}} \int_{-T / 2}^{T / 2}\left|\sum_{k_{j} \in \mathcal{K}} p_{k_{j}}\left(a_{k} e^{i \lambda_{k} t}+a_{-k} e^{-i \lambda_{k} t}\right)\right|^{2} \mathrm{~d} t \\
& \succsim \sum_{j=1}^{d} \sum_{k \in \mathcal{K}^{d}, \delta_{j} \leq\left|p_{k_{j}}\right|} A_{k}\left|p_{k_{j}}\right|^{2}-\sum_{j=1}^{d}\left(\frac{2 \pi}{T \gamma_{j}}\right)^{2} \sum_{k \in \mathcal{K}^{d}} A_{k}\left|p_{k_{j}}\right|^{2}=\sum_{k \in \mathcal{K}^{d}} A_{k} \sum_{j=1}^{d}\left(1_{\delta_{j} \leq\left|p_{k_{j}}\right|}-\left(\frac{2 \pi}{T \gamma_{j}}\right)^{2}\right)\left|p_{k_{j}}\right|^{2} .
\end{aligned}
$$

Since we notice that

$$
\sum_{j=1}^{d} 1_{\delta_{j} \leq \mid p_{k_{j}}}\left|p_{k_{j}}\right|^{2} \geq \max _{j=1, \ldots, d}\left|p_{k_{j}}\right|^{2} \geq \sum_{j=1}^{d}\left|\frac{p_{k_{j}}}{\gamma_{j}}\right|^{2} / \sum_{j=1}^{d} \frac{1}{\gamma_{j}^{2}}
$$

we finally get

$$
B \succsim\left(1 / \sum_{j=1}^{d} \frac{1}{\gamma_{j}^{2}}-\left(\frac{2 \pi}{T}\right)^{2}\right) \sum_{k \in \mathcal{K}^{d}} A_{k}\left(\sum_{j=1}^{d}\left|\frac{p_{k_{j}}}{\gamma_{j}}\right|^{2}\right) \geq\left(1 / \sum_{j=1}^{d} \frac{1}{\gamma_{j}^{2}}-\left(\frac{2 \pi}{T}\right)^{2}\right) \sum_{k \in \mathcal{K}^{d}} A_{k}\left(\sum_{j=1}^{d}\left|p_{k_{j}}\right|^{2}\right),
$$

which ends the proof of Theorem 2.1, from the assumption $T>2 \pi \sqrt{\sum_{i=1}^{d} \frac{1}{\gamma_{i}^{2}}}$ and since we can change the interval $[-T / 2, T / 2]$ into $[0, T]$ by a classical translation argument.

\section{Application for the proof of Proposition 1.1}

In order to prove Proposition 1.1 by using Theorem 2.1, we first express the inequality (2) in terms of the Fourier series (3). We have at first

$$
\begin{aligned}
& \int_{0}^{T} \int_{\Gamma}\left|\partial_{\nu} u(t, x)\right|^{2} \mathrm{~d} \Gamma \mathrm{d} \mathrm{t}=\sum_{\mathrm{j}=1}^{\mathrm{N}} \int_{0}^{\mathrm{T}} \int_{\mathrm{a}_{1}}^{\mathrm{b}_{1}} \ldots \int_{\mathrm{a}_{\mathrm{j}-1}}^{\mathrm{b}_{\mathrm{j}-1}} \int_{\mathrm{a}_{\mathrm{j}+1}}^{\mathrm{b}_{\mathrm{j}+1}} \cdots \int_{\mathrm{a}_{\mathrm{N}}}^{\mathrm{b}_{\mathrm{N}}} \\
&\left|\sum_{k \in\left(\mathbb{N}^{*}\right)^{N}} \frac{\pi k_{j}}{b_{j}-a_{j}}\left(c_{k} e^{i \omega_{k} t}+c_{-k} e^{-i \omega_{k} t}\right) \prod_{\ell=1, \ell \neq j}^{N} \sin \left(\frac{\pi k_{\ell}\left(x_{\ell}-a_{\ell}\right)}{b_{\ell}-a_{\ell}}\right)\right|^{2} \mathrm{~d} x_{1} \ldots \mathrm{d} x_{j-1} \mathrm{~d} x_{j+1} \mathrm{~d} x_{N} \mathrm{~d} t
\end{aligned}
$$

Due to the orthogonality of the family $\left(\sin \left(\frac{\pi k_{\ell}\left(x_{\ell}-a_{\ell}\right)}{b_{\ell}-a_{\ell}}\right)\right)_{k_{\ell} \geq 1}$ in $L^{2}\left(a_{\ell}, b_{\ell}\right)$, we get

$$
\int_{0}^{T} \int_{\Gamma}\left|\partial_{\nu} u(t, x)\right|^{2} \mathrm{~d} \Gamma \mathrm{d} \mathrm{t} \asymp \sum_{\mathrm{j}=1}^{\mathrm{N}} \sum_{\mathrm{k}_{1}, \ldots, \mathrm{k}_{\mathrm{j}-1}, \mathrm{k}_{\mathrm{j}+1}, \ldots, \mathrm{k}_{\mathrm{N}} \in \mathbb{N}^{*}} \int_{0}^{\mathrm{T}}\left|\sum_{\mathrm{k}_{\mathrm{j}} \in \mathbb{N}^{*}} \mathrm{k}_{\mathrm{j}}\left(\mathrm{c}_{\mathrm{k}} \mathrm{e}^{\mathrm{i} \omega_{\mathrm{k}} \mathrm{t}}+\mathrm{c}_{-\mathrm{k}} \mathrm{e}^{-\mathrm{i} \omega_{\mathrm{k}} \mathrm{t}}\right)\right|^{2} \mathrm{dt} .
$$


On the other hand, we have

$$
\left\|u_{0}\right\|_{H_{0}^{1}(\Omega)}^{2}+\left\|u_{1}\right\|_{L^{2}(\Omega)}^{2} \asymp \sum_{k \in\left(\mathbb{N}^{*}\right)^{N}}\left(\sum_{j=1}^{N}\left|k_{j}\right|^{2}\right)\left(\left|c_{k}\right|^{2}+\left|c_{-k}\right|^{2}\right) .
$$

We then apply Theorem 2.1: we take $d=N, \mathcal{K}=\mathbb{N}^{*}, \lambda_{k}=\omega_{k}$, for $k \in\left(\mathbb{N}^{*}\right)^{N}$ and $p_{\ell}=\ell$, for $\ell \in \mathbb{N}^{*}$. In order to check the gap assumption (4)-(5)-(6), for a fixed $j \in\{1, \ldots, N\}$, we thus consider $k=$ $\left(k_{1}, \ldots, k_{N}\right) \in\left(\mathbb{N}^{*}\right)^{N}$ and $k_{j}^{\prime} \in \mathbb{N}^{*}$, such that

$$
0<k_{i} \leq \max \left(k_{j}, k_{j}^{\prime}\right), i=1, \ldots, N, i \neq j,
$$

and we have to obtain (4)-(5), for a suitable $\gamma_{j}$. We compute

$$
\left|\omega_{k}-\omega_{k^{\prime}}\right|=\left|k_{j}-k_{j}^{\prime}\right| \frac{\pi^{2}}{\left(b_{j}-a_{j}\right)^{2}} \frac{\left|k_{j}+k_{j}^{\prime}\right|}{\sqrt{D+\left(\frac{k_{j} \pi}{b_{j}-a_{j}}\right)^{2}}+\sqrt{D+\left(\frac{k_{j}^{\prime} \pi}{b_{j}-a_{j}}\right)^{2}}},
$$

with $D=\sum_{i=1, i \neq j}^{N}\left(\frac{k_{i} \pi}{b_{i}-a_{i}}\right)^{2}$. We can suppose for instance that $k_{j} \leq k_{j}^{\prime}$ and thus have by using (13)

$$
\begin{array}{r}
\left|\omega_{k}-\omega_{k^{\prime}}\right| \geq\left|k_{j}-k_{j}^{\prime}\right| \frac{\pi^{2}}{\left(b_{j}-a_{j}\right)^{2}} \frac{\left|1+\left(k_{j} / k_{j}^{\prime}\right)\right|}{\sqrt{\sum_{i=1, i \neq j}^{d}\left(\frac{\pi}{b_{i}-a_{i}}\right)^{2}+\left(k_{j} / k_{j}^{\prime}\right)^{2} \frac{\pi^{2}}{\left(b_{j}-a_{j}\right)^{2}}}+\sqrt{\sum_{i=1}^{d}\left(\frac{\pi}{b_{i}-a_{i}}\right)^{2}}} \\
\geq\left|k_{j}-k_{j}^{\prime}\right| \frac{\pi^{2}}{\left(b_{j}-a_{j}\right)^{2}} \frac{1}{2 \sqrt{\sum_{i=1}^{d}\left(\frac{\pi}{b_{i}-a_{i}}\right)^{2}}} .
\end{array}
$$

On the other hand, we have $\left|\omega_{k}+\omega_{k^{\prime}}\right| \geq\left|k_{j}+k_{j}^{\prime}\right| \frac{\pi}{\left(b_{j}-a_{j}\right)}$. We get thus (4)-(5) by taking

$$
\gamma_{j}=\frac{\pi^{2}}{\left(b_{j}-a_{j}\right)^{2}} \frac{1}{2 \sqrt{\sum_{i=1}^{d}\left(\frac{\pi}{b_{i}-a_{i}}\right)^{2}}}<\frac{\pi}{\left(b_{j}-a_{j}\right)} .
$$

From (11) and (12), we finally get (2) with $T>2 \pi \sqrt{\sum_{j=1}^{N} \gamma_{j}^{2}}=4 \sqrt{\sum_{j=1}^{N}\left(b_{j}-a_{j}\right)^{4}} \sqrt{\sum_{j=1}^{N}\left(b_{j}-a_{j}\right)^{-2}}$, which ends the proof of Proposition 1.1. Note that if we could take $\gamma_{j}=\frac{\pi}{\left(b_{j}-a_{j}\right)}$, we would obtain the optimal time $T_{0}=2 \sqrt{\sum_{i=1}^{N}\left(b_{i}-a_{i}\right)^{2}}$.

\section{References}

[1] P. Loreti and M. Mehrenberger, An Ingham type proof for a bigrid observability theorem, ESAIM COCV 14 (3), (2008), 604-631.

[2] V. Komornik, On the exact internal controllability of a Petrowsky system, J. Math. Pures Appl. (9) 71 (1992), 331-342.

[3] V. Komornik, Exact Controllability and Stabilization-The Multiplier Method, John Wiley and Masson, Chicester and Paris, 1994.

[4] V. Komornik and P. Loreti, Fourier Series in Control Theory, Springer, New York, 2005.

[5] P. Loreti and V. Valente, Partial exact controllability for spherical membranes, SIAM J. Control Optim. 35 (1997), 641-653.

[6] K. Ramdani, T. Takahashi, G. Tenenbaum and M. Tucsnak, A spectral approach for the exact observability of infinitedimensional systems with skew-adjoint generator, J. Funct. Anal. 226 (1) (2005), 193-229. 